\title{
Map Calculation of the Shapley-Shubik Voting Powers: An Example of the European Economic Community
}

\author{
Ali Muhammad Ali Rushdi* \\ Department of Electrical and Computer Engineering, Faculty of Engineering \\ King Abdulaziz University, P. O. Box 80204, Jeddah 21589, Saudi Arabia \\ E-mail: arushdi@kau.edu.sa \\ ${ }^{*}$ Corresponding author \\ Omar Mohammed Ba-Rukab \\ Department of Information Technology, Faculty of Computing and Information Technology \\ King Abdulaziz University P. O. Box 344, Rabigh, 21911, Saudi Arabia \\ E-mail: obarukab@kau.edu.sa
}

(Received November 4, 2016; Accepted November 19, 2016)

\begin{abstract}
The paper utilizes the Karnaugh map in a pedagogical calculation of the Shapley-Shubik index of voting power, which is perhaps the most prominent such index for managerial, political, and engineering voting systems. The Karnaugh map has features that allow it to readily implement this calculation, namely: (a) it can conveniently represent pseudo-switching functions of the form $g=B_{2}^{n} \rightarrow R$, (b) it can immediately detect the number of orderings in going from one of its configurations (cells) to another, and (c) it visually identifies a pair of configurations that is critical $w . r$. $t$. a certain voter, which are a primitive winning coalition of which this voter is a member and a corresponding primitive losing coalition that results when this voter defects. The paper offers a detailed description of these three features and of the associated calculations using the celebrated system of the European Economic Community as a running example.
\end{abstract}

Keywords - Shapley-Shubik index, Voting power, Karnaugh map, Pseudo-switching function, Ordering, Winning coalition.

\section{Introduction}

The Shapley-Shubik Index (SSI) is perhaps the most well-known "cardinal notion of power." It is a quantitative measure of how much a member of a yes-no voting system exercises "control over outcomes" of that system. This notion of power applies to any yes-no system (and not to just a weighted voting system). This paper offers a tutorial exposition of how the Shapley-Shubik index can be calculated efficiently with the aid of the Karnaugh map from the perspective of a famous voting system, namely that of the European Economic Community. The Karnaugh map is nothing but a truth table representation of a switching function (a two-valued Boolean function) (Lee, 1978; Muroga, 1979; Rushdi, 1997; Rushdi and Ghaleb, 2015). However, it enjoys the double advantage that it is two-dimensional rather than one-dimensional and that its cells possess adjacency properties that are easily visualized by the human user. The Karnaugh map procedure reported herein is a simple, easy to visualize and comprehend, and amenable to very fast hand calculation; i.e., it enjoys features that allow it to play a role supplementary to that of advanced and sophisticated algorithms (See, e.g., Klinz and Woeginger, 2005). The map procedure also gives its user a glimpse of the combinatorial nature of the problem of computing a power index, and hence grants him/her an intuitive feeling for the "intractable" nature of this problem (Matsui and Matsui, 2001).

The organization of the rest of this paper is as follows. Section 2 reviews some pertinent nomenclature, while Sec. 3 introduces our running example which is the voting system of the European Economic Community (EEC). Sections 4-6 demonstrate clearly why the Karnaugh map 
International Journal of Mathematical, Engineering and Management Sciences

Vol. 2, No. 1, 17-29, 2017

https://dx.doi.org/10.33889/IJMEMS.2017.2.1-002

is most suitable for the required task. Section 4 shows how the Karnaugh map is conveniently used to represent a pseudo switching function, and Sec. 5 demonstrates the map's capability of counting the orderings of going from a configuration to another, while Sec. 6 utilizes the map in identifying pairs of configurations that are critical w. r. t. a certain voter. Section 7 accomplishes the required task of computing the SSI of the EEC members, while Sec. 8 concludes the paper.

\section{Nomenclature}

A voting system: A set of rules that specifies how voters choose among several candidates or alternatives.

A yes-no voting system: A voting system where the choice is between two alternatives, or typically there is a simple question of adopting a single alternative (a bill, resolution or amendment) against the status quo (Taylor and Pacelli, 2008).

A coalition: Any set of yes-voters. If the debated alternative is upheld (i.e., if the bill, resolution or amendment is passed) the coalition is winning; otherwise it is losing. The coalition is empty if no voter belongs to it and it is the grand coalition if all voters belong to it (Nurmi, 1997; Taylor and Pacelli, 2008).

The pivotal voter for a given ordering of voters: Suppose we add voters one by one according to the given ordering, starting with the losing empty coalition and ending with the winning grand coalition, then the pivotal voter for this ordering is the one whose joining converts this growing coalition from a non-winning one to a winning one (Taylor and Pacelli, 2008).

The total Shapley-Shubik index of a voter (TSSI(P)): The total Shapley-Shubik index is the number of orderings for which the vote $\mathrm{P}$ is the pivotal voter. If the number of voters is $\mathrm{n}$, then the set $V$ of all voters is of cardinality $|V|=\mathrm{n}$, and the total number of possible orderings of the set $V$ is n!, (Shapley and Shubik, 1954; Felsenthal and Machover, 1996; Taylor and Pacelli, 2008; Wallis, 2014), i.e.,

$\sum_{i=1}^{n} \operatorname{TSSI}\left(P_{i}\right)=n !$

The Shapley-Shubik index of a voter $\left(\operatorname{SSI}\left(P_{i}\right)\right)$ : The Shapley-Shubik index is the number between zero and one that represents the fraction of possible orderings for which the voter $P$ is the pivotal player, i.e.,

$\operatorname{SSI}\left(P_{i}\right)=\operatorname{TSSI}\left(P_{i}\right) / n !$

Hence, condition (1a) can be cast in the normalized form, in which the sum of SS indices for all voters is unity, i.e.,

$\sum_{i=1}^{n} \operatorname{SSI}\left(P_{i}\right)=1$

\section{Running (Recurring) Example of a Voting System}

In this section, we describe a yes-no weighted voting system that we use as a running (recurring) example throughout this paper. This is the example of the European Economic Community, a predecessor of the contemporary European Union (Taylor and Pacelli, 2008). In 1958, the European Economic Community was established as a weighted voting system of six countries, namely: 
International Journal of Mathematical, Engineering and Management Sciences

Vol. 2, No. 1, 17-29, 2017

https://dx.doi.org/10.33889/IJMEMS.2017.2.1-002

France (F), Germany (G), Italy (I), Belgium (B), the Netherlands (N), and Luxembourg (L). The three large countries (France, Germany, and Italy) were given four votes each, while the mediumsized Belgium and the Netherlands were assigned two votes each and tiny Luxembourg was granted just a single vote. Passage of a decision required at least twelve of the seventeen possible votes. This system can be described by a coherent threshold switching function $g(\boldsymbol{X})$ (Muroga, 1971; Rushdi, 1990; Rushdi and Alturki, 2015; Alturki and Rushdi, 2016), such that

$\{g(\boldsymbol{X})=1\} \Leftrightarrow\left\{\boldsymbol{W}^{T} \boldsymbol{X}=4 F+4 G+4 I+2 B+2 N+L \geq 12\right\}$

where $\boldsymbol{X}=\left[\begin{array}{llllll}F & G & I & B & N & L\end{array}\right]^{T}$ and $\boldsymbol{W}=\left[\begin{array}{llllll}4 & 4 & 4 & 2 & 2 & 1\end{array}\right]^{T}$ are a vector of switching (two-valued Boolean) variables, and another of corresponding weights, respectively. Due to symmetry considerations we note that

$\operatorname{SSI}(F)=\operatorname{SSI}(G)=\operatorname{SSI}(I)$

$\operatorname{SSI}(B)=\operatorname{SSI}(N)$

Hence, we will be content in the sequel to study the SSI of either France (F), Germany (G) or Italy (I) (say SSI(F)) and the SSI of either Belgium (B) or the Netherlands (N) (say SSI(B)). Using (4a) and $(4 b)$ in $(1 b)$, we obtain

$\boldsymbol{W}^{\boldsymbol{T}} \boldsymbol{X}=3 \operatorname{SSI}(F)+2 \operatorname{SSI}(B)+\operatorname{SSI}(L)=1$

At first glance, it seems that this voting system has deliberately favored Luxemburg by assigning it a relative weight that far exceeds what is warranted by its relative size (population, area, wealth, etc.,) when compared with the other countries. We will see shortly that this is not really the case, and that Luxemburg is, in fact, a dummy voter of no power at all since there is no single ordering for which Luxemburg is a pivotal voter. There is no winning coalition of which Luxemburg is a member such that it becomes losing if Luxemburg defects from it. Likewise, no losing coalition that does not include Luxemburg becomes winning when Luxemburg joins it. The question of "fair" allocation of weights remained a matter of concern when the EEC outgrew gradually into the current European Union (Felsenthal and Machover, 1997a; Laruelle and Widgrèn, 1998; Bilbao et al., 2002). This question is also important for other international organizations such as the International Monetary Fund (Leech, 2002).

\section{Construction of a Karnaugh map for a Pseudo-Switching Function}

A notable advantage of the Karnaugh map is that it can be conveniently used to represent a pseudoswitching function $g=B_{2}^{n} \rightarrow R_{2}$ (Rushdi, 1987a; 1987b; 1988; 1990b; Rushdi and Alturki, 2015; Rushdi and Ghaleb, 2016). Note that the domain of $g$ is similar to that of a switching function $f=$ $B_{2}^{n} \rightarrow B_{2}$, but the range of $g$ is the real line, while that of $f$ is the switching set $\{0,1\}$. Therefore, the Karnaugh map for $g$ has the same structure or input variables as the map for $f$ has, but its entries or values of its output variable are reals and not necessarily restricted to the values 0 and 1 . There are at least two methods for constructing a Karnaugh map of the pseudo-switching function

$g(\boldsymbol{X})=\boldsymbol{W}^{\boldsymbol{T}} \boldsymbol{X}=\sum_{i=1}^{n} W_{i} X_{i}$

This Karnaugh map has $\mathrm{n}$ variables $\boldsymbol{X}(1 \leq i \leq n)$ and hence comprises $2^{n}$ cells. In our first method for constructing this map, we obtain its entries gradually via the following procedure: 
International Journal of Mathematical, Engineering and Management Sciences

Vol. 2, No. 1, 17-29, 2017

https://dx.doi.org/10.33889/IJMEMS.2017.2.1-002

- Initialize all map entries as zeros;

- For $i:=1$ to $n$ do begin

- Construct the loop $X_{i}$ covering the asserted region of $X_{i}$ which is the half map that constitutes $2^{n-1}$ cells;

- Augment the current entry in each of the $2^{n-1}$ cells of the $X_{i}$ loop by the real value $W_{i}$. End.

Fig.1 illustrates the application of the above procedure in constructing a Karnaugh map for the sum of weighted votes

$g(F, G, I, B, N, L)=W_{F} F+W_{G} G+W_{I} I+W_{B} B+W_{N} N+W_{L} L$

of the European Economic Community, where the switching variables F, G, I, B, N, L stand for France, Germany, Italy, Britain, the Netherlands, and Luxemburg, with corresponding weights

$W_{F}=W_{G}=W_{I}=4, W_{B}=W_{N}=2$ and $W_{L}=1$.

The final result of applying the above procedure is shown in Fig. 2, which displays a 6-variable map with symbolic entries. Fig. 2 also demonstrates our equivalent second method for constructing the required map. We start by entering a 0 at the top leftmost cell, which is called the all-0 cell since it is outside the asserted region of each of the six variables (and also called the configuration of the empty coalition). Now, we move around the map cells. As we go from a particular map cell to an adjacent cell, then we will be crossing from one region of a single variable $X_{i}$ to its opposite region without changing region for the rest of the variables. If we are moving upwards, i.e., if we are crossing from the non-asserted region of $X_{i}$ to its asserted region then the cell entry should be increased by $W_{i}$. If , we happen to move downwards, i.e., to cross from the asserted region of $X_{i}$ to its non asserted region, then we decrease the cell entry by $W_{i}$.

Fig. 3 is a replica of Fig. 2, in which the pertinent weights are assigned their numerical values. Here, an entry that equals or exceeds the threshold value of 12 is highlighted in bold and its configuration is called a primitive winning coalition (PWC). Other cells of the map contain nonbold entries of values less than 12 and are said to represent primitive non-winning or losing coalitions. 
International Journal of Mathematical, Engineering and Management Sciences

Vol. 2, No. 1, 17-29, 2017

https://dx.doi.org/10.33889/IJMEMS.2017.2.1-002

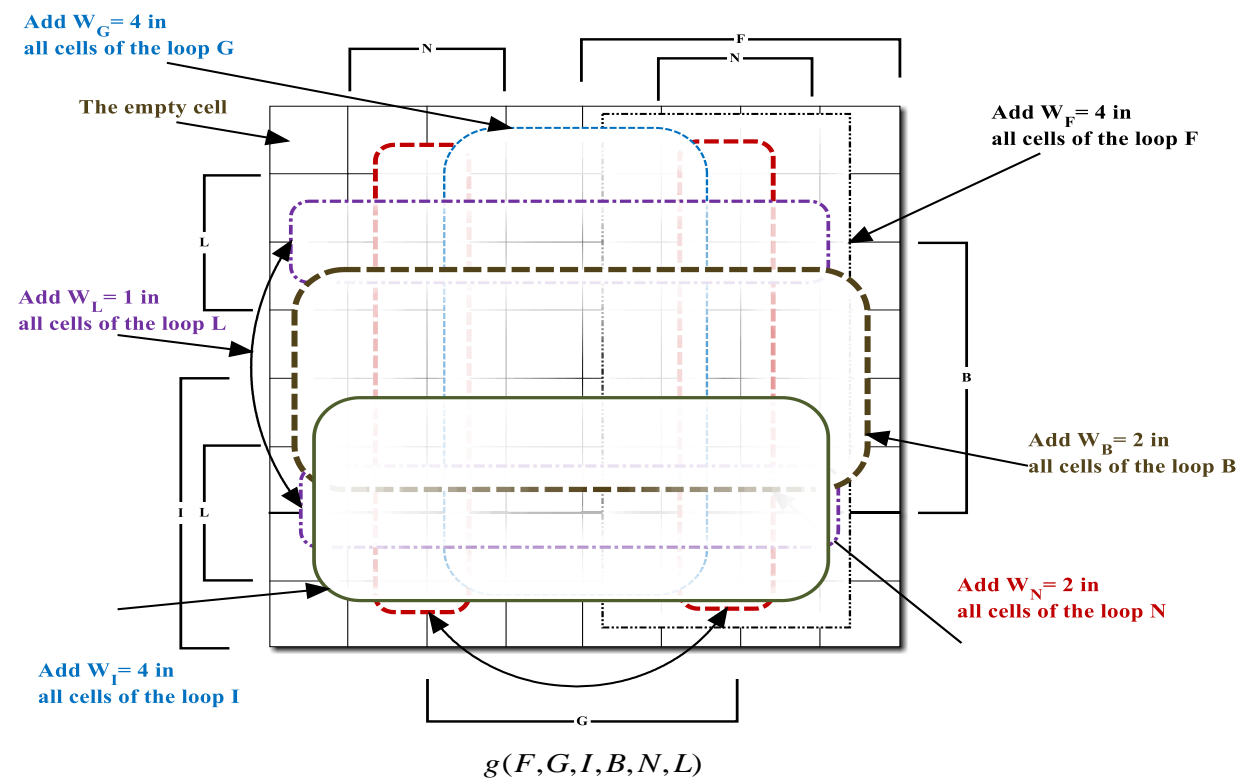

Fig. 1. Use of half-map loops to enter the weights in the pseudo switching function $g(F, G, I, B, N, L)=$ $W_{F} F+W_{G} G+W_{I} I+W_{B} B+W_{N} N+W_{L} L$

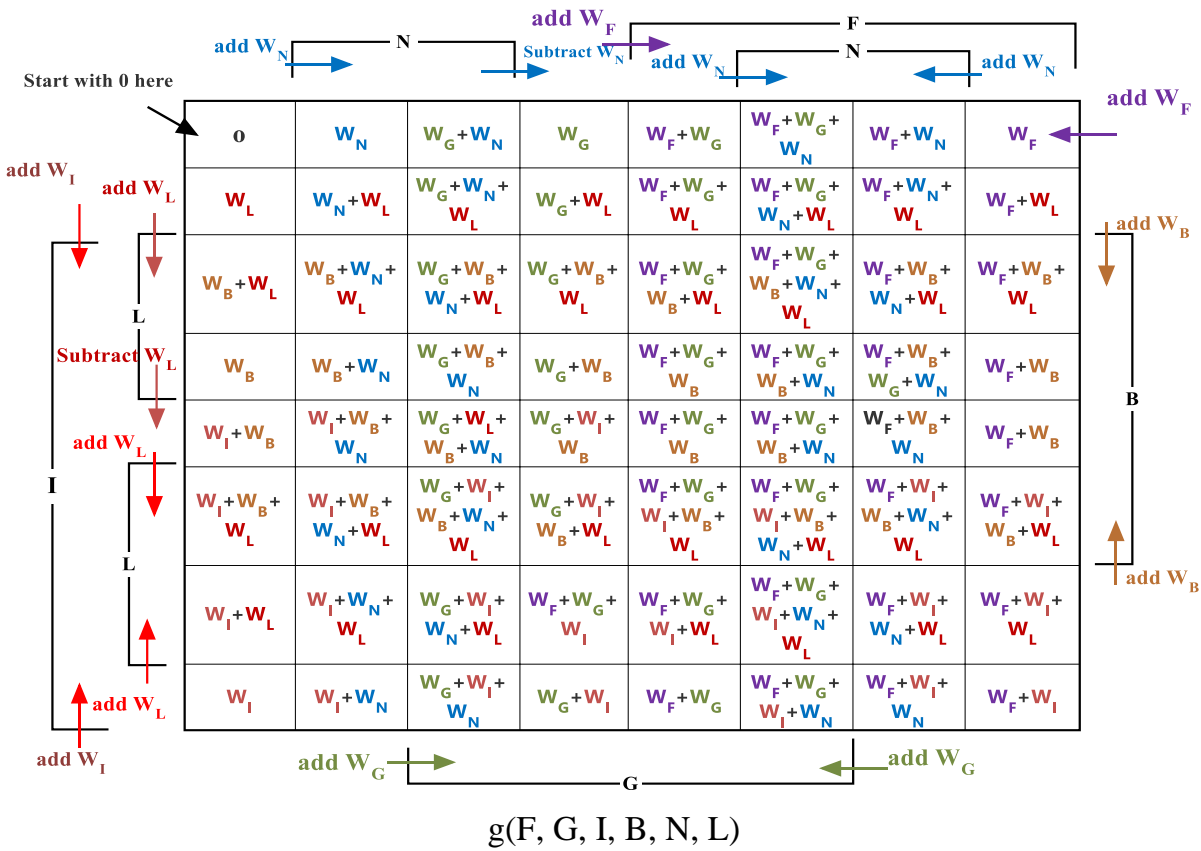

Fig. 2. The pseudo-switching function $g$ with symbolic entries representing sums of pertinent weights. Here, the half map labeled by a certain variable is called the asserted region of the variable, while the other half of the map is called its non-asserted region. 
International Journal of Mathematical, Engineering and Management Sciences

Vol. 2, No. 1, 17-29, 2017

https://dx.doi.org/10.33889/IJMEMS.2017.2.1-002

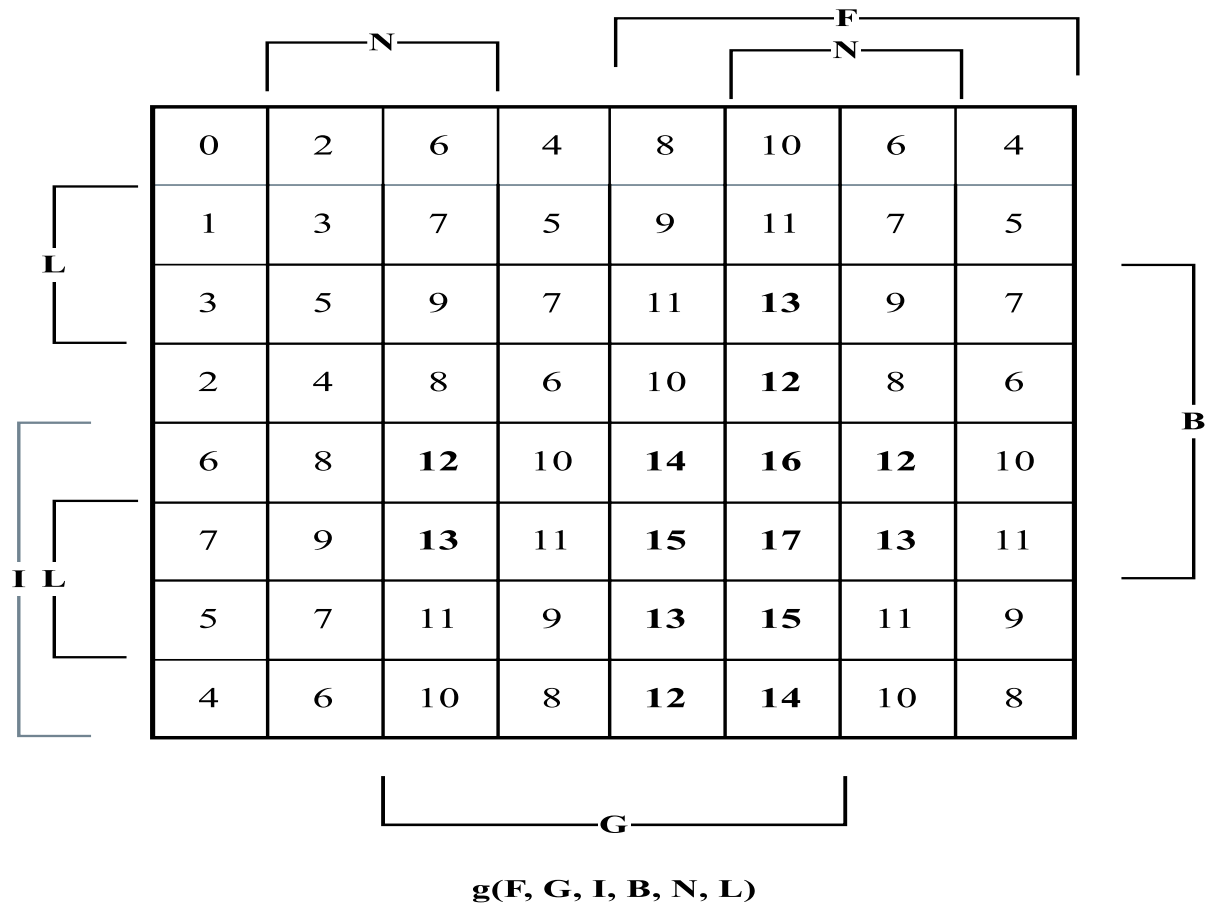

Fig. 3. The pseudo-switching function $g$ with numerical entries representing the sum of the pertinent weights. An entry exceeding or equal to the threshold of 12 is shown bold and occupies a cell of a primitive winning coalition. Other cells represent primitive losing coalitions

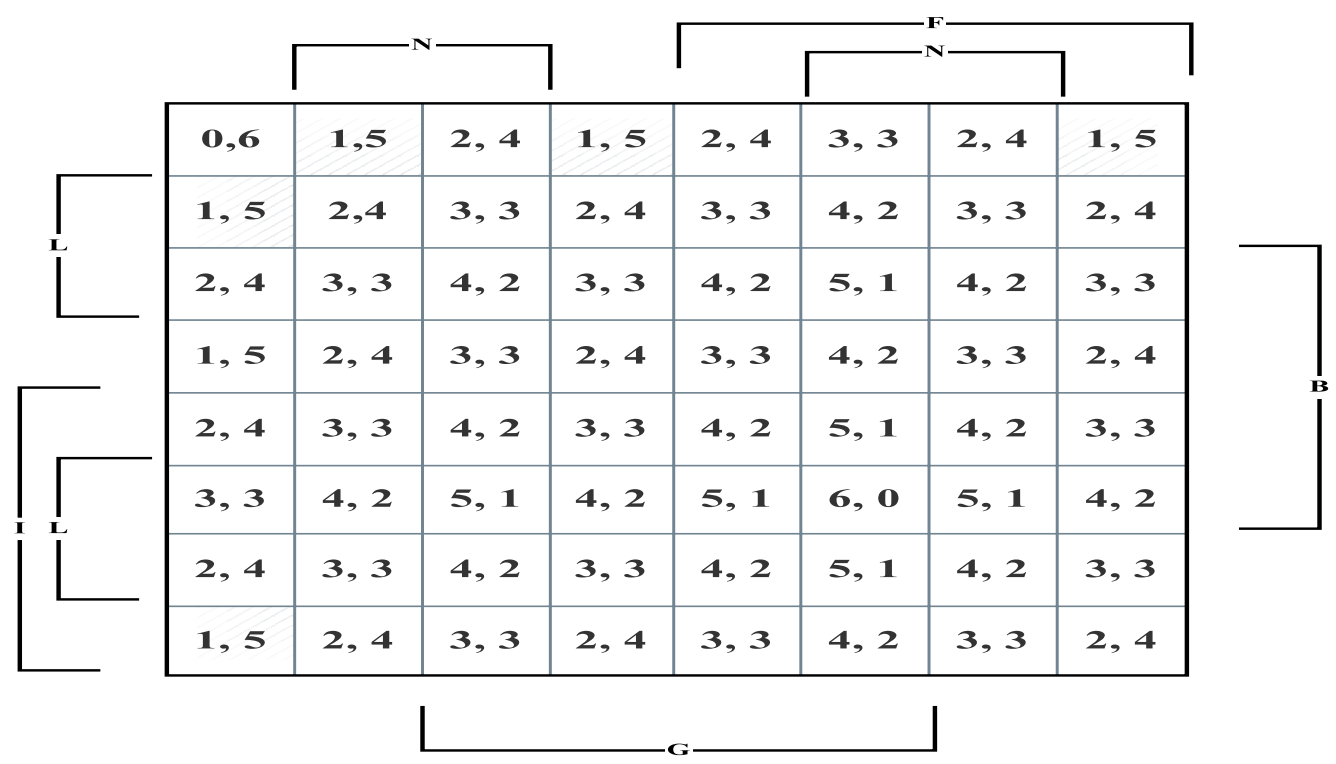

Fig. 4. A six-variable Karnaugh map with every cell characterized by its Hamming distance to the all-0 cell (the empty coalition) and its distance to the all-1 cell (the grand coalition). The sum of these two distances is always six. 
International Journal of Mathematical, Engineering and Management Sciences

Vol. 2, No. 1, 17-29, 2017

https://dx.doi.org/10.33889/IJMEMS.2017.2.1-002

\section{The Number of Orderings for Moving Between Two Map Cells}

Another prominent merit of the Karnaugh map is that it offers a natural way of detecting the number of orderings for moving between two map cells. Let the Hamming distance $\mathrm{d}\left(C_{1}, C_{2}\right)$ between two map cells $C_{1}$ and $C_{2}$ be the number of their differing variable regions (the number of differing variable polarities in their minterm expansions, or the number of bit differences in their binary number representations) (Hamming, 1980; Rushdi and Ghaleb, 2016). For example, if $\mathrm{C}_{1}$ is the top leftmost cell of the map it is represented by the minterm $\bar{F} \bar{G} \bar{I} \bar{B} \bar{N} \bar{L}$ or the binary number 000000 (that is why it is named the all-0 cell). If $C_{2}$ is the bottom rightmost cell, then it is represented by the minterm $F \bar{G} I \bar{B} \bar{N} \bar{L}$ or the binary number 101000 . The Hamming distance between these two cells is

$$
\begin{gathered}
\mathrm{d}\left(C_{1}, C_{2}\right)=(0 \oplus 1)+(0 \oplus 0)+(0 \oplus 1)+(0 \oplus 0)+(0 \oplus 0)+(0 \oplus 0) \\
=1+0+1+0+0+0=2
\end{gathered}
$$

The number of orderings $\mathrm{O}\left(C_{1}, C_{2}\right)$ for moving between the two cells is the factorial of the Hamming distance between them, namely

$\mathrm{O}\left(C_{1}, C_{2}\right)=\left(\mathrm{d}\left(C_{1}, C_{2}\right) !\right)$

For our example cells, this number is $2 !=2$. In fact, the map clearly shows that we can move from $C_{1}$ to $C_{2}$ either via $F \bar{G} \bar{I} \bar{B} \bar{N} \bar{L}$ (100000) or via $\bar{F} \bar{G} I \bar{B} \bar{N} \bar{L}$ (001000). Similarly the distance from $C_{1}$ to the cell $C_{3}$ given by $F \bar{G} I \bar{B} N \bar{L}$ (101010) is 3 and the number of orderings $\mathrm{O}\left(C_{1}, C_{3}\right)$ is 6 , namely

$$
\begin{aligned}
& 000000 \rightarrow \mathbf{1 0 0 0 0 0} \rightarrow \mathbf{1 0 1 0 0 0} \rightarrow 101010 \\
& 000000 \rightarrow \mathbf{1 0 0 0 0 0} \rightarrow \mathbf{1 0 0 0 1 0} \rightarrow 101010 \\
& 000000 \rightarrow 001000 \rightarrow \mathbf{1 0 1 0 0 0} \rightarrow 101010 \\
& 000000 \rightarrow 001000 \rightarrow 001010 \rightarrow 101010 \\
& 000000 \rightarrow 000010 \rightarrow \mathbf{1 0 0 0 1 0} \rightarrow 101010 \\
& 000000 \rightarrow 000010 \rightarrow 001010 \rightarrow 101010
\end{aligned}
$$

We stress herein that by "orderings" we refer to shortest paths or minimal transitions. We do not allow paths that cross variable boundaries more than once, i.e., in opposite directions. We are particularly interested in the all-0 cell, and its dual the all-1 cell (the cell F G I B N L or 1111111 1) and in particular cells that we call critical with respect to a certain voter. These critical cells occur in pairs: a cell representing a primitive losing coalition (PLC) within the non-asserted region of the pertinent voter, of which this voter is not a member, and an adjacent cell (within the asserted region of this voter) that represents a primitive winning coalition (PWC), which results from the sole change that the pertinent voter joins the aforementioned PLC. In particular, we need information about the Hamming distance from the all-0 cell to a PLC-cell and the Hamming distance from a PWC-cell to the all-1 cell. Such information is tabulated in (and might be memorized from) the Karnaugh map of Fig. 4. This map can be prepared once and for all. It need not be prepared afresh every time its information is needed. Note that the numbers entered in the Karnaugh map of Fig. 4 are frequently used to characterize symmetric switching functions (Lee, 1987; Rushdi, 1986; 1993; 2010). 
International Journal of Mathematical, Engineering and Management Sciences

Vol. 2, No. 1, 17-29, 2017

https://dx.doi.org/10.33889/IJMEMS.2017.2.1-002

\section{Identification of a Pair of Coalitions that is Critical to a Certain Voter}

The third advantage of using the Karnaugh map in calculating the SSIs is now explained. The map allows an immediate visual inspection of every pair of coalitions that are critical to any voter, simply by identifying the border of the asserted region for this voter and looking for a PLC that becomes a PWC upon transition to its image across this border. Fig. 5 highlights the F-boundary (the boundary between the asserted and non-asserted regions of F), and consequently recognizes critical pairs across the F-boundary. In such critical pairs a transition takes place from a PLC to a PWC across the F-boundary. Fig. 6 does the same for the B-boundary, while Fig. 7 demonstrates that there are no critical pairs across the L-boundary.

\section{Map Calculations of the SSIs}

Voter $\mathrm{P}$ is a pivotal one when there exists a pair of primitive coalitions that are exactly the same except that (a) P does not belong to the first and belongs to the second, and (b) the first is losing and the second is winning. We call these two coalitions critical $w . r . t$. voter P. Let pairs of such coalitions be indexed by $i_{P}$, where $1 \leq i_{P} \leq I_{P}$. Hence, the total value for SSI (P), called TSSI(P) is the summation over $i_{P}$ of the number of orderings from the empty coalition to the grand coalition through the pair of $i_{P}$ coalitions that is critical to $\mathrm{P}$, i.e.,

$$
\begin{aligned}
\operatorname{TSSI}(P)= & \sum_{i_{P}=1}^{I_{P}} O\left(\text { all }-0, \text { all }-1 \mid \text { through losing } i_{P} \text { and winning } i_{P}\right)= \\
& \sum_{i_{P}=1}^{I_{P}} O\left(\text { all }-0, \text { losing } i_{P}\right) \times O\left(\text { losing } i_{P}-\text { winning } i_{P}\right) \times \\
= & \sum_{i_{P}=1}^{I_{P}}\left(\left(d\left(\text { all }-0, \text { losing } i_{P}\right)\right) !\right) \times 1 \times\left(\left(d\left(\text { winning } i_{P}, \text { all }-1\right)\right) !\right)= \\
& \sum_{i_{P}=1}^{I_{P}}\left(d\left(\text { empty coalition }, \text { losing } i_{P}\right) !\right) \times\left(d\left(\text { winning } i_{P}, \text { grand coalition }\right) !\right)
\end{aligned}
$$

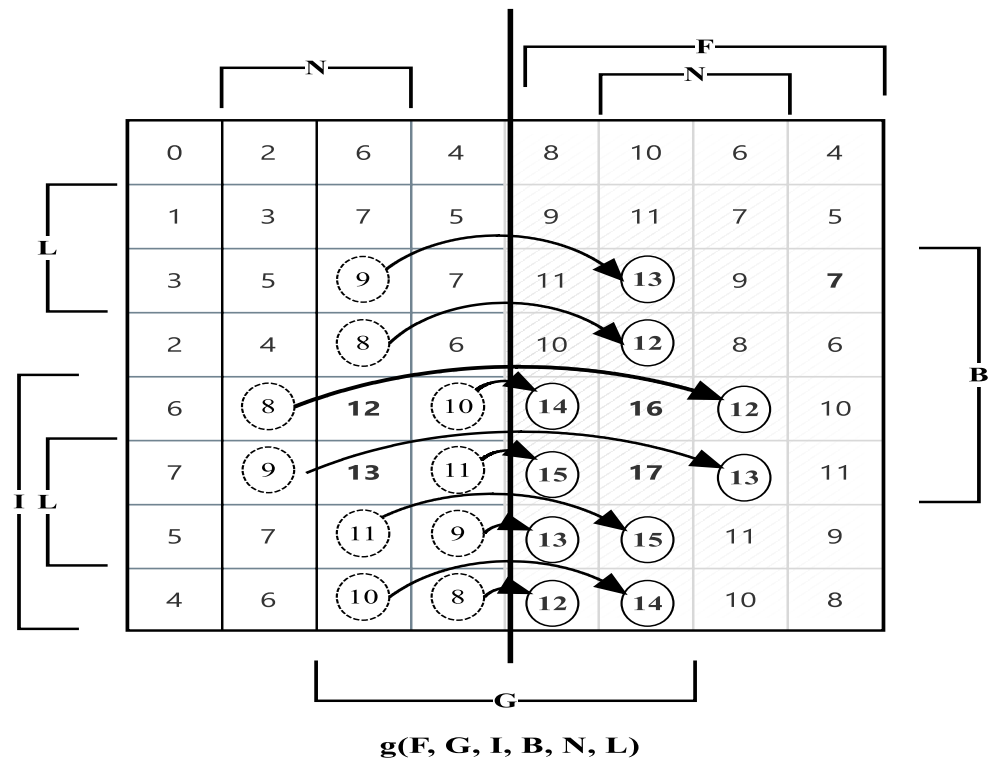

Fig. 5. A pseudo-switching function representing the sum of weighted votes. A pair of critical coalitions $w$. $r$. $t$. F are identified by an arrow going from a critical primitive losing coalition with a non-bold entry within a dotted ellipse to the corresponding critical primitive winning coalition (with bold entry within a continuous ellipse) 
International Journal of Mathematical, Engineering and Management Sciences

Vol. 2, No. 1, 17-29, 2017

https://dx.doi.org/10.33889/IJMEMS.2017.2.1-002

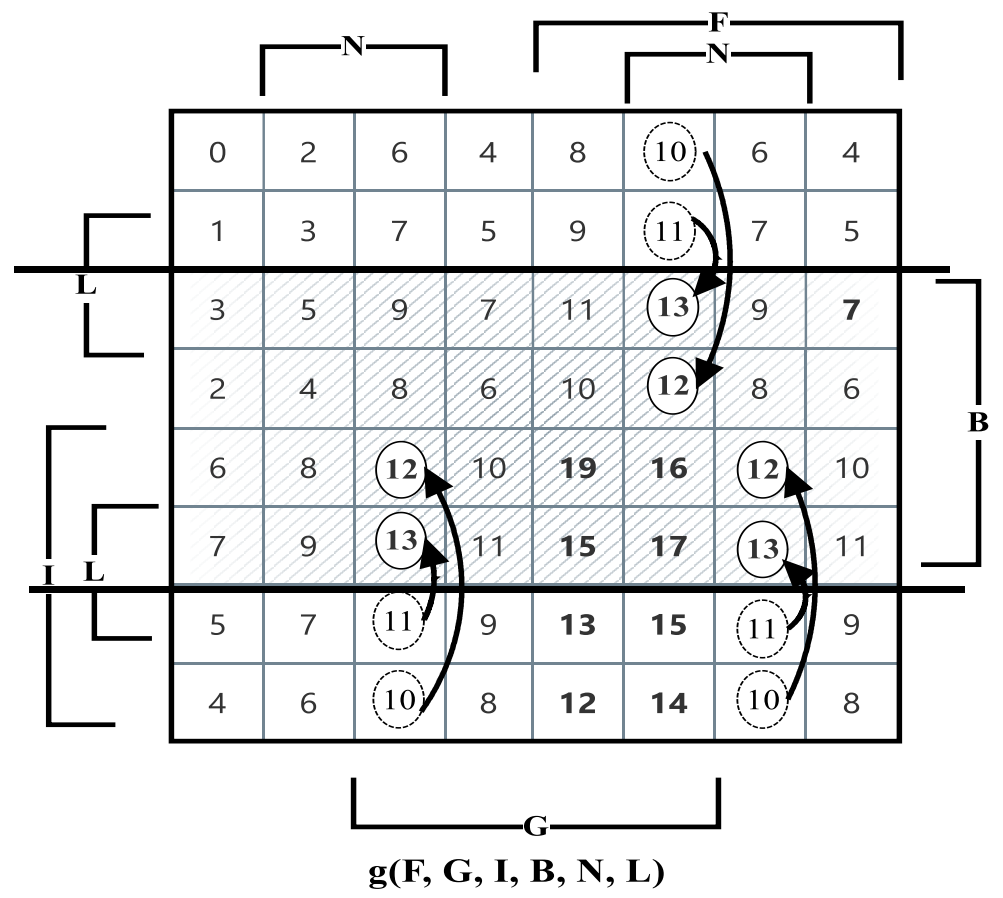

Fig. 6. A pseudo-switching function representing the sum of weighted votes. A pair of critical coalitions $w$. $r . t$. B are identified by an arrow going from a critical primitive losing coalition with a non-bold entry within a dotted ellipse to the corresponding critical primitive winning coalition (with bold entry within a continuous ellipse)

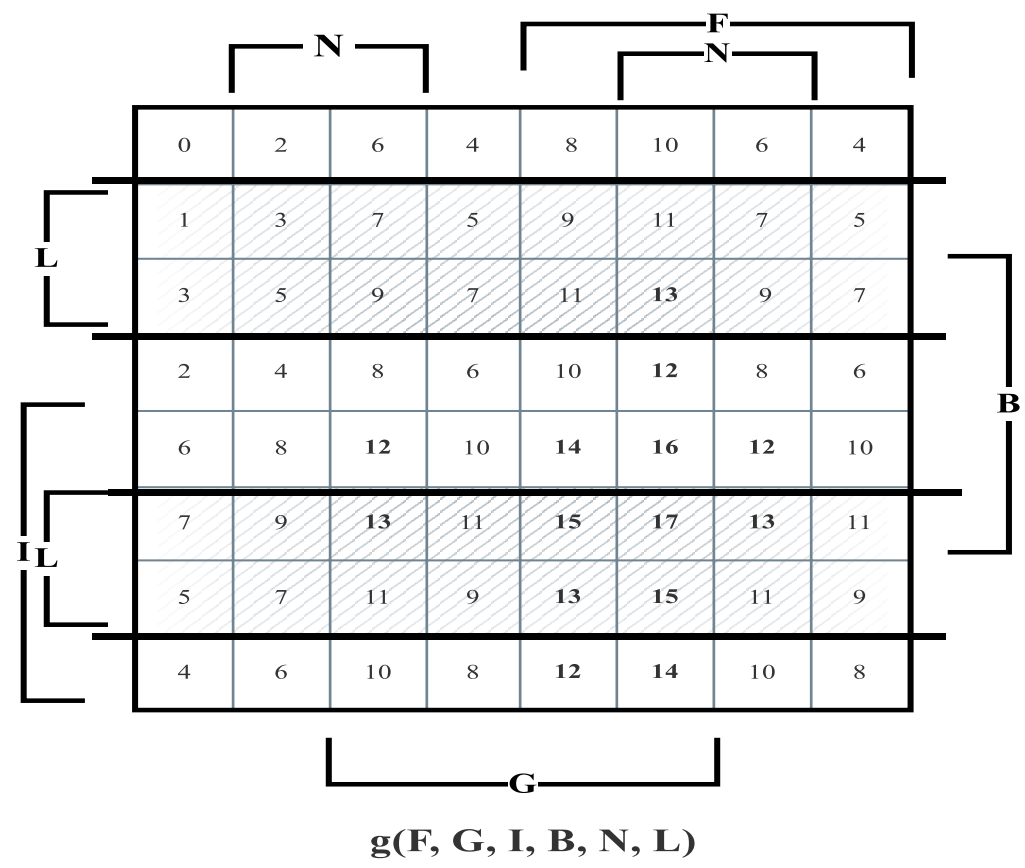

Fig. 7. A pseudo-switching function representing the sum of weighted votes, showing that there is no primitive winning coalition of which $\mathrm{L}$ is a member, and her defection is critical 
International Journal of Mathematical, Engineering and Management Sciences

Vol. 2, No. 1, 17-29, 2017

https://dx.doi.org/10.33889/IJMEMS.2017.2.1-002

Using the above equation (10), and aided visually by Figs. 8 and 9, we obtain

TSSI $(\mathrm{F})=\mathrm{TSSI}(\mathrm{G})=\mathrm{TSSI}(\mathrm{I})=4(4 !)(1 !)+5(3 !)(2 !)+1(3 !)(2 !)$

$$
=96+60+12=168
$$

$\operatorname{TSSI}(\mathrm{B})=\operatorname{TSSI}(\mathrm{N})=3(4 !)(1 !)+3(3 !)(2 !)=72+36=108$

By virtue of Fig. 7, there are no transitions across the L-boundary from a PLC to a PWC. Hence,

$\operatorname{TSSI}(\mathrm{L})=0$

These TSSI values should be normalized by their sum which is

$3(168)+2(108)+1(0)=720$

which equals (6!), as expected from (1a). Finally, we obtain

$$
\begin{aligned}
& \operatorname{SSI}(\mathrm{F})=\operatorname{SSI}(\mathrm{G})=\operatorname{SSI}(\mathrm{I})=\frac{168}{720}=\frac{14}{60} \\
& \operatorname{SSI}(\mathrm{B})=\operatorname{SSI}(\mathrm{N})=\frac{108}{720}=\frac{9}{60} \\
& \operatorname{SSI}(L)=0
\end{aligned}
$$

In passing, we note that we used (1a) (or equivalently, we could have used (1c)) only as a check. Alternatively, we could have made some significant computational saving if we made an early observation of the fact that $\mathrm{SSI}(\mathrm{L})=0$, and then computed $\mathrm{SSI}(\mathrm{F})$ only and relied on (1c) to express SSI(B) in terms of SSI(F).

We also observe that $I_{P}$ denotes the total number of pairs of primitive coalitions that are critical $w$. r. t. voter $P$, and hence $I_{P}$ can be identified as TBI(P) the total Banzhaf Index of $P$, which is another prominent index of voting power (Taylor and Pacelli, 2008), viz.

$\operatorname{TBI}(P)=I_{P}$

The total Shapley-Shubik Index $\operatorname{TSSI}(P)$ is more sophisticated and perhaps more informative then the total Banzhaf Index $\operatorname{TBI}(P)$. If and only if one of these two indexes is zero, the other is also zero and the concerned voter is a dummy one as it has zero power, i.e.,

$\{P$ has zero power $\} \Leftrightarrow\{\operatorname{TSSI}(P)=0\} \Leftrightarrow\{\operatorname{TBI}(P)=0\}$

While TBI $(P)$ makes a simple count of the total number of critical pairs of primitive coalitions, SSI $(P)$ makes a weighted count of the same number. In our running example, the values of Total Banzhaf Indices are

$$
\begin{aligned}
& \operatorname{TBI}(\mathrm{F})=\operatorname{TBI}(\mathrm{G})=\operatorname{TBI}(\mathrm{I})=10 \\
& \operatorname{TBI}(\mathrm{B})=\operatorname{TBI}(\mathrm{N})=6 \\
& \operatorname{TBI}(\mathrm{L})=0
\end{aligned}
$$


International Journal of Mathematical, Engineering and Management Sciences

Vol. 2, No. 1, 17-29, 2017

https://dx.doi.org/10.33889/IJMEMS.2017.2.1-002

\section{Conclusions and Future Work}

This paper strived to make the most of the Karnaugh map in a straightforward visually-aided calculation of the Shapley-Shubik Index of voting power. The strengths of the Karnaugh map that enhanced its suitability for such a calculation were highlighted and demonstrated. The Karnaugh map can be used also in computing other indices of voting power such as the Banzhaf Index.

The current analysis dealt with yes-no voting, and hence ignored the possibility of abstentions. Ignoring abstentions, Taylor and Pacelli (2008) proved that the UN Security council without abstentions is a weighted voting system with a weight of 7 for a permanent member, a weight of 1 for a non-permanent member, and a threshold of 39 . For this system, the only winning coalition is $P_{1} P_{2} P_{3} P_{4} P_{5} S y\left(\left\{\begin{array}{ll}4 & 10\end{array}\right\}, N\right)$, where $P_{i}(1 \leq i \leq 5)$ represents a UN permanent member while $\operatorname{Sy}(\{4 \ldots 10\}, \boldsymbol{N})$ is the symmetric switching function of 10 variables $\boldsymbol{N}$ representing the nonpermanent members of the council (with characteristic set $\{4 \ldots 10\}=\{4,5,6,7,8,9,10\}$. In this model, each of the non-permanent members has zero power while the permanent members are equally powerful. Reality is not that severe for non-permanent members, since permanent members might occasionally abstain (albeit on solely trivial or non-critical issues). A better model that warrants on further future exploration might account for abstentions by using ternary logic (Flsenthal and Machover, 1997b).

The current calculations have not attempted to benefit of symmetries (apart from predicting that certain SSIs are equal). It might be useful to find certain shortcuts in the calculations via symmetry.

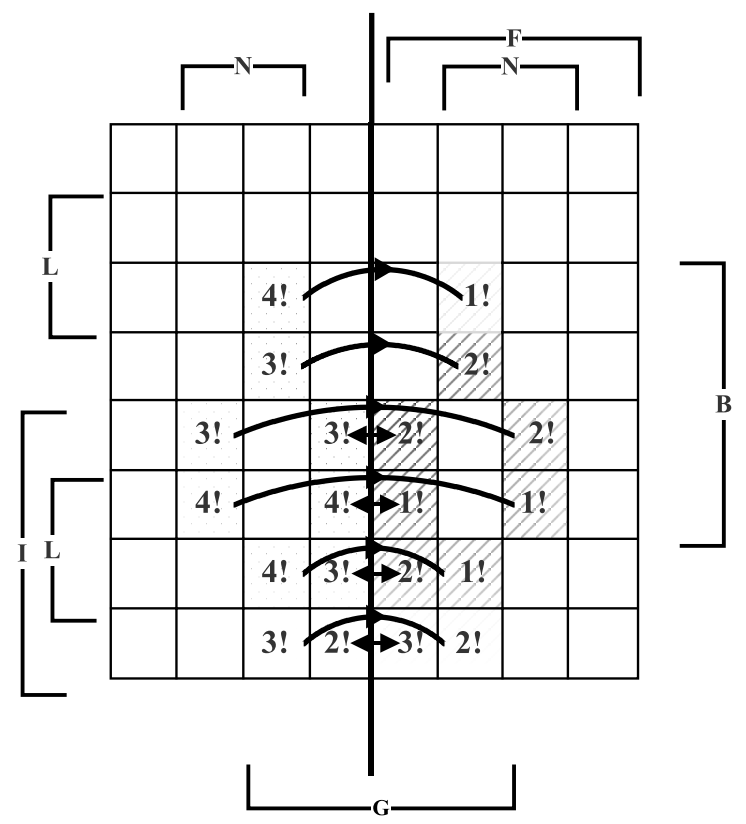

Fig. 8. Transitions from primitive losing coalitions (dotted) to their images across the F-boundary to corresponding primitive winning ones (hatched). These are the transitions in which $\mathrm{F}$ is pivotal (critical). Other transitions across the F-boundary are either losing-losing transitions or winning-winning transitions. In a cell for a primitive losing coalition we write the number of direct ways it can be reached from the all-0 cell (the factorial of that cell's distance from the all- 0 cell). In a cell for a primitive winning coalition, we write the number of direct ways the all 1-cell can be reached from that cell (the factorial of that cell's distance to the all-1 cell) 
International Journal of Mathematical, Engineering and Management Sciences

Vol. 2, No. 1, 17-29, 2017

https://dx.doi.org/10.33889/IJMEMS.2017.2.1-002

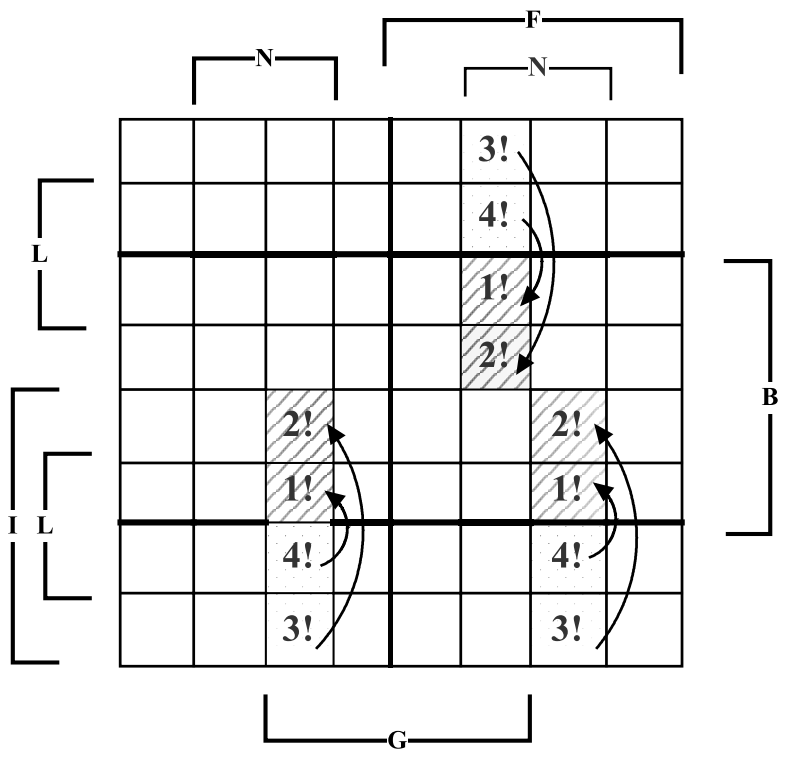

Fig. 9. Transitions similar to those in Fig. 8, but now w. r. t. the B-boundary.

\section{References}

Alturki, A. M. \& Rushdi, A. M. A., (2016). Weighted voting systems: A threshold-boolean perspective, Journal of Engineering Research, 4(1), 125-143.

Bilbao, J. M., Fernandez, J. R., Jiménez, N., \& Lopez, J. J. (2002). Voting power in the European Union enlargement. European Journal of Operational Research, 143(1), 181-196.

Felsenthal, D. S., \& Machover, M. (1996). Alternative forms of the Shapley value and the Shapley-Shubik index. Public choice, 87(3-4), 315-318.

Felsenthal, D. S., \& Machover, M. (1997a). The weighted voting rule in the EU's Council of Ministers, 19581995: Intentions and outcomes. Electoral Studies, 16(1), 33-47.

Felsenthal, D. S., \& Machover, M. (1997b). Ternary voting games. International Journal of Game Theory, 26(3), 335-351.

Hamming, R. W. (1980). Coding and Information Theory. Prentice-Hall, Englewood Cliffs, NJ, USA.

Klinz, B., \& Woeginger, G. J. (2005). Faster algorithms for computing power indices in weighted voting games. Mathematical Social Sciences, 49(1), 111-116.

Laruelle, A., \& Widgrén, M. (1998). Is the allocation of voting power among EU states fair?. Public Choice, 94(3-4), 317-339.

Lee, S. C. (1978). Modern Switching Theory and Digital Design, Prentice-Hall, Englewood Cliffs, New Jersey, NJ, USA.

Leech, D. (2002). Voting power in the governance of the International Monetary Fund. Annals of Operations Research, 109(1-4), 375-397

Matsui, Y., \& Matsui, T. (2001). NP-completeness for calculating power indices of weighted majority games. Theoretical Computer Science, 263(1), 305-310.

Muroga, S. (1971). Threshold Logic and Its Applications, Wiley-Interscience, New York, NY, USA. 
International Journal of Mathematical, Engineering and Management Sciences

Vol. 2, No. 1, 17-29, 2017

https://dx.doi.org/10.33889/IJMEMS.2017.2.1-002

Muroga, S. (1979). Logic Design and Switching Theory, John Wiley \& Sons, New York, NY, USA.

Nurmi, H., (1997). On power indices and minimal winning coalitions, Control and Cybernetics, 26: 609-612.

Rushdi, A. M. (1986). Utilization of symmetric switching functions in the computation of k-out-of-n system reliability. Microelectronics and Reliability, 26(5), 973-987.

Rushdi, A. M. (1987a). Probabilistic performance indices of power generation and transmission systems. In Proceedings of the First Symposium on Electric Power Systems in Fast Developing Countries, Riyadh, Saudi Arabia (pp. 247-254).

Rushdi, A. M. (1987b). Capacity function-preserving star-delta transformations in flow networks. Reliability engineering, 19(1), 49-58.

Rushdi, A. M. (1988). Performance indexes of a telecommunication network. IEEE Transactions on Reliability, R-37(1), 57-64.

Rushdi, A. M. (1990a). Threshold systems and their reliability. Microelectronics and Reliability, 30(2): 299312.

Rushdi, A. M. (1990b). Star-delta transformations of bidirectional branches in probabilistic flow networks. Microelectronics and Reliability, 30(3), 525-535.

Rushdi, A. M. (1993). Reliability of k-out-of-n systems. In K. B. Misra (Editor), New Trends in System Reliability Evaluation. Elsevier, Amsterdam, 16, 185-227.

Rushdi, A. M. (2010). Partially-redundant systems: Examples, reliability, and life expectancy. International Magazine on Advances in Computer Science and Telecommunications, 1(1), 1-13.

Rushdi, A. M. A. \& Alturki, A. M. (2015). Reliability of coherent threshold systems. Journal of Applied Science, 15(3): 431-443.

Rushdi, A. M. A., \& Ghaleb, F. A. M. (2015). The Walsh spectrum and the real transform of a switching function: a review with a Karnaugh-map perspective. Journal of Qassim University: Engineering and Computer Sciences, 7(2), 73-112.

Rushdi, A. M. A., \& Ghaleb, F. A. M. (2016). A tutorial exposition of semi-tensor products of matrices with a stress on their representation of Boolean functions. Journal of King Abdulaziz University: FCIT, 5(1), $3-41$.

Rushdi, A. M., (1997). Karnaugh map, Encyclopedia of Mathematics, Supplement Volume I, M. Hazewinkel (Editor), Boston, Kluwer Academic Publishers, pp. 327-328. Available at http://eom.springer.de/K/k110040.html.

Shapley, L. S., \& Shubik, M. (1954). A method for evaluating the distribution of power in a committee system. American Political Science Review, 48(03), 787-792.

Taylor, A. D., \& Pacelli, A. M. (2008). Mathematics and Politics: Strategy, Voting, Power, and Proof. Springer Science \& Business Media.

Wallis, W. D. (2014). The Mathematics of Elections and Voting. Springer International Publishing Switzerland. 